\title{
Optical Beam Steering using 2-D Surface Acoustic Wave Actuator
}

\author{
Takashi Shigematsu ${ }^{1}$, Takayuki Iseki ${ }^{2}$, Mikio Okumura ${ }^{2}$, Takashi Sugawara ${ }^{2}$, and Minoru K. Kurosawa ${ }^{1}$ \\ ${ }^{1}$ Department of Advanced Applied Electronics, Tokyo Institute of Technology, JAPAN \\ ${ }^{2}$ Technology Development Division, Victor Company of Japan, Limited, JAPAN \\ e-mail: sgmt@ieee.org
}

\begin{abstract}
Steering angle over 20 degrees two-axis optical beam steering prototype that employs a mirror on a two-axis gimbal is developed, which takes advantage of two-dimensional planner surface acoustic wave actuator. For the actuation of 20 degrees of tilt angle, $10 \mathrm{~ms}$ duration of driving power input is required for actuation, then additional $10 \mathrm{~ms}$ for mirror structure's damping produced complete angle holding. Note that any power supply is unnecessary to hold the angle. The resolution of the angle was 0.003 degree because of high-resolution ability of the actuator. The beam steering prototype showed $10^{4}$ dynamic range of angle positioning ability.
\end{abstract}

Keywords: Beam Steering, Ultrasonic Motor, Planner Motor, Surface Acoustic Wave, MEMS

\section{INTRODUCTION}

Two-axis beam steering devices have been utilized for optical switches such in networking systems, e.g. optical routers. The kinds of optical components seem to be particularly promising applications for MEMS (Micro Electro Mechanical Systems) devices owing to the advantageous ability of miniaturization/integration. A great variety of optical MEMS devices thus have been proposed.

Optical MEMS devices usually use electrostatic forces for actuation of mirrors, which is advantageous for miniaturization and integration, however that imposes a limitation in increasing the tilt angle. Thus it seems to have difficulty in applying MEMS mirrors to the equipments that require large steering angle, e.g. bar-code readers or optical scanners. There are, however, a certain degrees of demands to reduce occupied volume of such devices so that the two-axis micro beam steering devices would be developed.

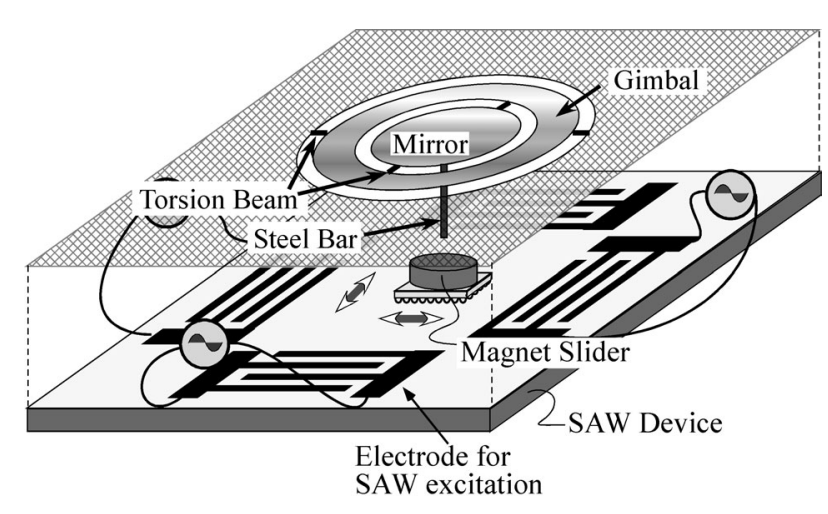

Fig. 1. Schema of the beam steering prototype.

A surface acoustic wave (SAW) actuator is an ultrasonic linear motor, which utilizes piezoelectric crystal for a stator $[1,2]$ and a silicon substrate element for a slider [2,3]. Both substrates were widely used in commercial and their fabrication processes are common MEMS techniques, so that the high quality and miniature feature fabrication process are guaranteed. To date, the minimum size of the actuator has been reported was $3 \times 12.5 \times 2.5 \mathrm{~mm}^{3}$ [4].

The operating principle based on frictional force against traveling SAW (ultrasonic) wave affords up to cm-level long stroke of the slider's motion. The friction drive principle, on the other hand, provides self-holding force due to the static frictional force when the driving power is off. In addition, the actuator could easily expand to two-dimensional planner motor [5]. Therefore, implementing the actuator for a mirror actuation would achieve large operation angle two-axis beam steering along with self-holding function. Moreover, nanometerlevel positioning ability of the actuator $[3,5]$ and up to $1.5 \mathrm{~m} / \mathrm{s}$ high-speed mobility [6] would provide precise positioning and high-speed scanning of reflected beam.

We have proposed a beam steering prototype in which the SAW actuator steers a mirror on a gimbal [7], however performances have not yet been evaluated. In this report, performances of the prototype are reported.

\section{TRANSDUCERS'05}

The 13th International Conference on Solid-State Sensors, Actuators and Microsystems, Seoul, Korea, June 5-9, 2005 

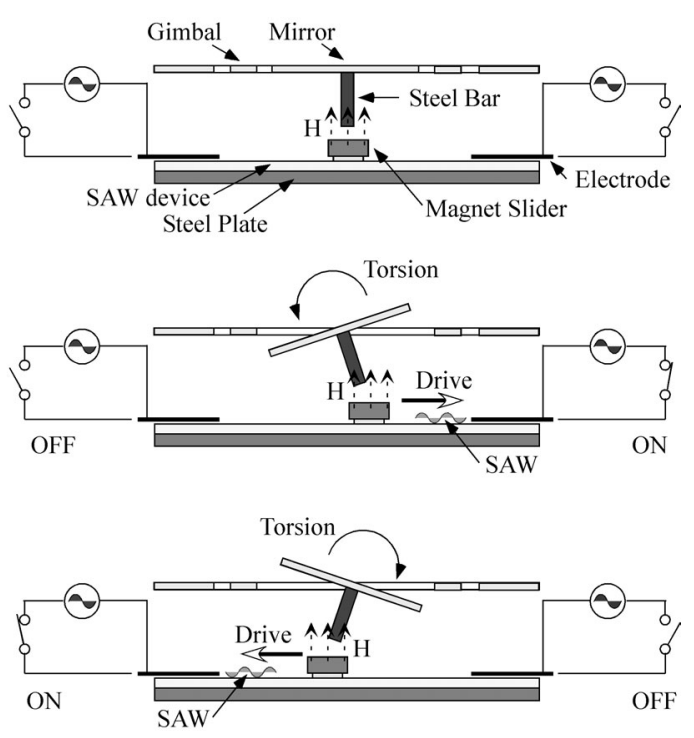

Fig. 2. Operating principle of mirror steering.

\section{PRINCIPLE}

A proposed two-axis beam steering prototype is made of two components: one is a gimbal-supported mirror that reflects optical beam, and the other is a SAW actuator that steers the mirror. The structure of the prototype is illustrated in Fig. 1. The SAW actuator is placed underneath the mirror at a certain distance. The actuator drives a magnet slider two dimensionally. At the center of the mirror's bottom side, a steel bar is attached, which follows the magnet by magnetic force. Consequently, the mirror is steered in two-axis by means of the displacement of the magnet slider.

Detailed driving principle is illustrated in Fig. 2. While the driving voltage is not applied, the magnet slider stands still by the frictional holding force and the mirror keeps the angle with the use of magnetic force between the magnet and the steel bar. When the driving voltage is applied to the right-side electrode (in the figure), the SAW propagates to the left. The frictional force against the SAW drives the magnet slider to the right. The free end of the steel bar simultaneously follows the magnet, which tilts the mirror in the counterclockwise direction. Likewise, the voltage input to the left side electrode tilts the mirror in the clockwise direction. The series of actuation, namely the voltage input to a pair of electrodes, steers the mirror in one-axis. The additional actuation using the other pair of the

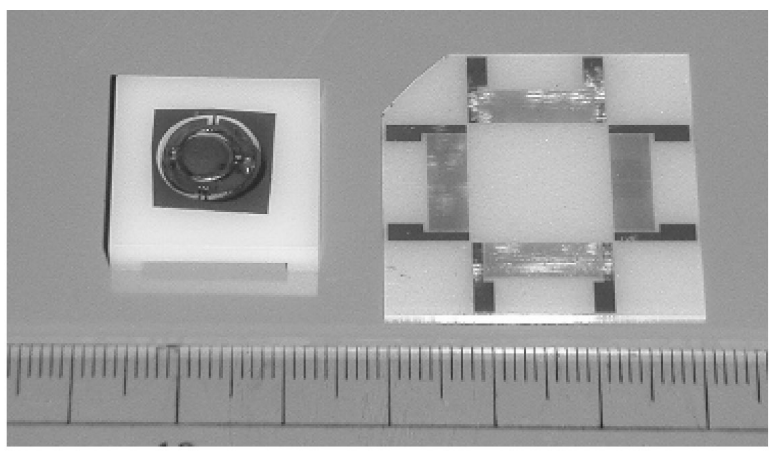

Fig.3. The mirror on gimbal and the SAW device.

electrodes in the orthogonal direction, therefore, makes possible the two-axis steering of the mirror.

\section{EXPERIMENTAL SETUP}

A gimbal-supported mirror structure was made of a polyimide sheet, which was fabricated by RIE. The diameters of the inner and the outer mirrors were 5 and 9 $\mathrm{mm}$, respectively. On the mirror surface, a reflector made of a silicon substrate was attached, which was fabricated by RIE as well. On the other side of the mirror, a $\phi 1 \times 3$ $\mathrm{mm}^{3}$ steel bar was glued.

A SAW device was made of $\mathrm{LiNbO}_{3}$ piezoelectric crystal substrate. The dimensions of the device were $31 \times 31 \times 0.5 \mathrm{~mm}^{3}$. At each end of the device, electrodes for SAW excitation were vacuum deposited. The electrode materials were chromium and aluminum. The driving frequency was approximately $20 \mathrm{MHz}$. A photograph of the mirror and the SAW device is indicated in Fig. 3.

The magnet slider was composed of a $\phi 3 \times 1.5 \mathrm{~mm}^{3}$ neodymium magnet and a $2 \times 2 \times 0.5 \mathrm{~mm}^{3}$ silicon slider, where they were glued together. The magnet slider totally weighted $0.078 \mathrm{~g}$. The magnet was not only used for the mirror actuation but produced normal force to the frictional surface, i.e., the magnet was magnetically attracted to a steel plate underneath the stator. Since the frictional force operates the SAW actuator, a certain amount of the normal force is necessary.

The silicon slider was made of a square plate of silicon substrate. The silicon slider had microlens projections on its frictional surface; the projections were $5 \mu \mathrm{m}$ in diameter, $2 \mu \mathrm{m}$ in height and arranged at $50-\mu \mathrm{m}$ pitch. The microlens projections were fabricated by means of thermal reflow of resist resin and RIE. 


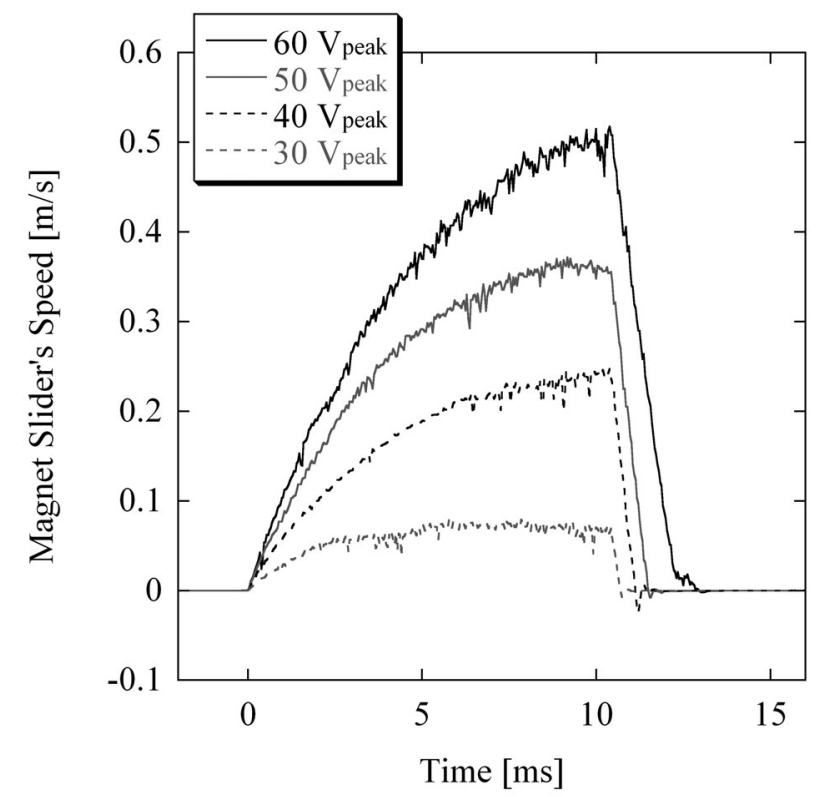

Fig. 4. Transient responses of the magnet slider.

\section{TRANSIENT RESPONSES}

The transient responses of the magnet slider are depicted in Fig. 4. A laser Doppler velocimeter measured the speed responses. The driving voltage was applied from $0 \mathrm{~ms}$ to approximately $10 \mathrm{~ms}$. As indicated in Fig.4, maximum speed of $0.5 \mathrm{~m} / \mathrm{s}$ was recorded on the condition of $60 \mathrm{~V}_{\text {peak }}$ driving voltage. The minimum driving voltage required for the actuation was $30 \mathrm{~V}_{\text {peak }}$; on the condition the magnet slider traveled at the speed of $0.05 \mathrm{~m} / \mathrm{s}$.

In the same way, by applying $50 \mathrm{~V}_{\text {peak }}$ driving voltage from $0 \mathrm{~ms}$ to $10 \mathrm{~ms}$, the transient response of the displacement of the magnet slider and the tilt angle of the mirror are indicated in Fig. 5. The gimbal structure was placed on the direction that only the inside of the mirror could tilt as illustrated in the figure. The laser Doppler interferometer measured the displacement of the inside mirror's edge, then simple trigonometric function calculation resulted the tilt angle.

As shown in the figure, $10 \mathrm{~ms}$ duration of the driving voltage input produced approximately $3 \mathrm{~mm}$ displacement of the magnet slider: the slider was perfectly stationary after the driving voltage turned off. In the meanwhile, the mirror followed the magnet slider's motion with a lag, where it tilted in the counterclockwise direction in the figure. The mirror
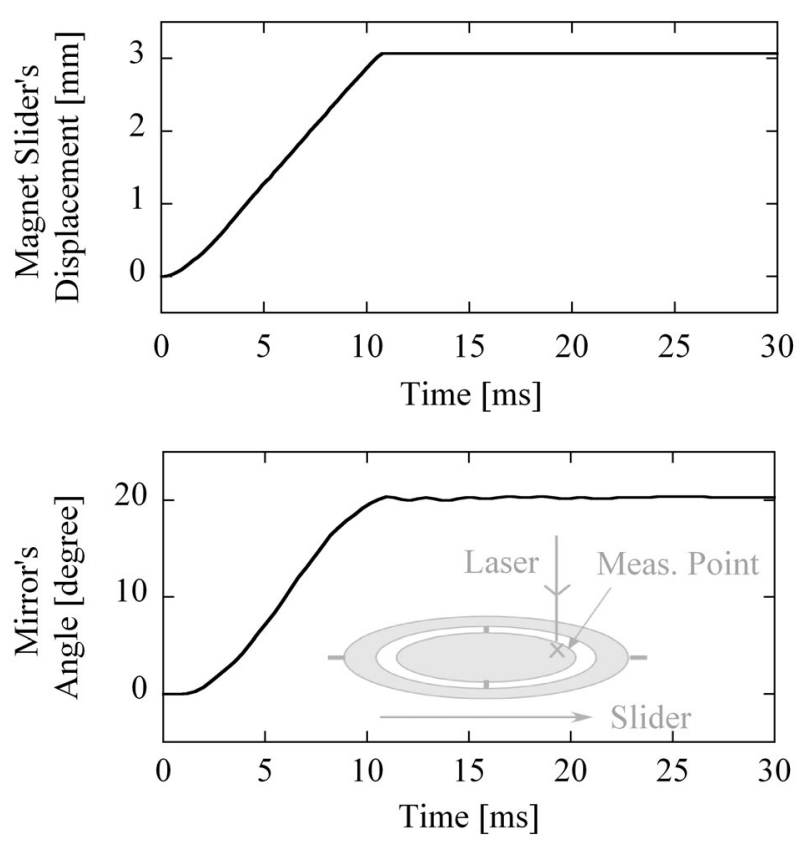

Fig. 5. Transient responses of the magnet slider on the SAW device and the mirror; the driving voltage was $50 \mathrm{~V}_{\text {peak }}$ and the driving duration was $10 \mathrm{~ms}$.

tilted approximately 20 degrees, when the slider stopped. It took additional $10 \mathrm{~ms}$ for the mirror to be stand still owing to the damping of the gimbal structure. The structure, then, completely held the tilt angle.

\section{TILT ANGLE}

The tilt angle in relation to the driving duration was investigated on the condition of $50 \mathrm{~V}_{\text {peak }}$ driving voltage. The experimental results are indicated in Fig. 6 together with the displacement of the magnet slider. The gimbal structure was placed on the direction that only the inside of the mirror could tilt. The tilt angle increased linearly with the displacement of the magnet slider. The resolution of the tilt angle depended on the stability of the gimbal structure. We could identify minimum 0.003 degree of the tilt angle. The fluctuation of the gimbal structure was on that level. The tilt angle of 0.003 degree corresponded to the displacement of $100 \mathrm{~nm}$ at the edge of the mirror. This prototype exhibited high-resolution angle positioning ability. The dynamic range of the tilt angle was on the order of $10^{4}$.

A step angle positioning, approximately 0.05 -degree step, is indicated in Fig. 7 as an example of the high 


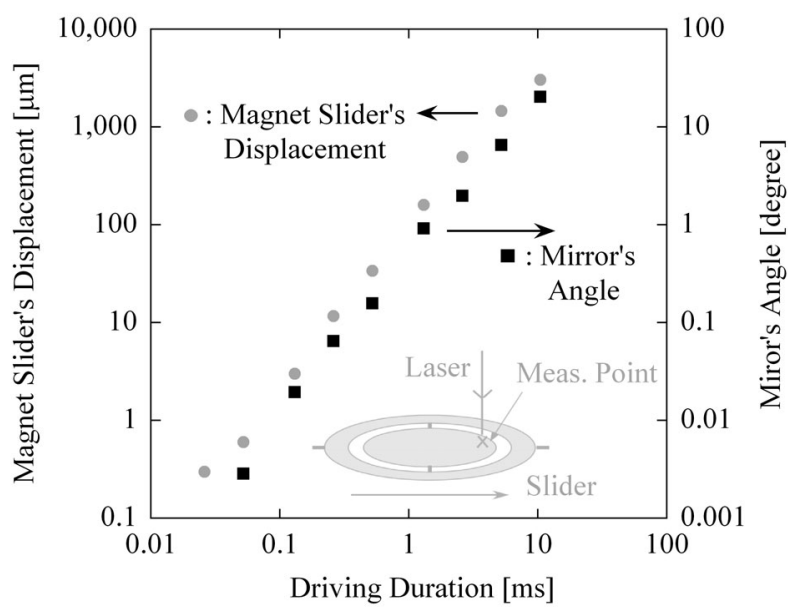

Fig. 6. The tilt angle of the mirror in relation to the driving duration: it plotted together with the displacement of the magnet slider. The driving voltage was $50 \mathrm{~V}_{\text {peak }}$.

resolution positioning of the prototype. The driving conditions were $50 \mathrm{~V}_{\text {peak }}$ driving voltage, $0.25 \mathrm{~ms}$ voltage applying duration, and $50 \mathrm{~ms}$ step period. At the time of $0.5 \mathrm{~s}$, the driving electrode was switched to the other one in the opposite side. The measurement of the mirror's displacement near the hinge of the gimbal was also indicated in the figure using the same scale. The mirror near the hinge was motionless during the step tilting motion of the mirror.

\section{SUMMARY}

The working principle and performances of the two-axis beam steering utilizing the SAW actuator is reported. The operating principle based on ultrasonic frictional drive and magnetic force produced the self-holding of the angle without power consumption, which is advantageous to the applications such infrequent switching equipments. The prototype demonstrated the 20 degrees of the steering range accompanied by 0.003 degree of resolution. Namely, the dynamic range was on the order of $10^{4}$.

\section{Acknowledgements}

This work was supported by the Grant-in-aid for Research Fellowships of the Japan Society for the Promotion of Science for Young Scientists.
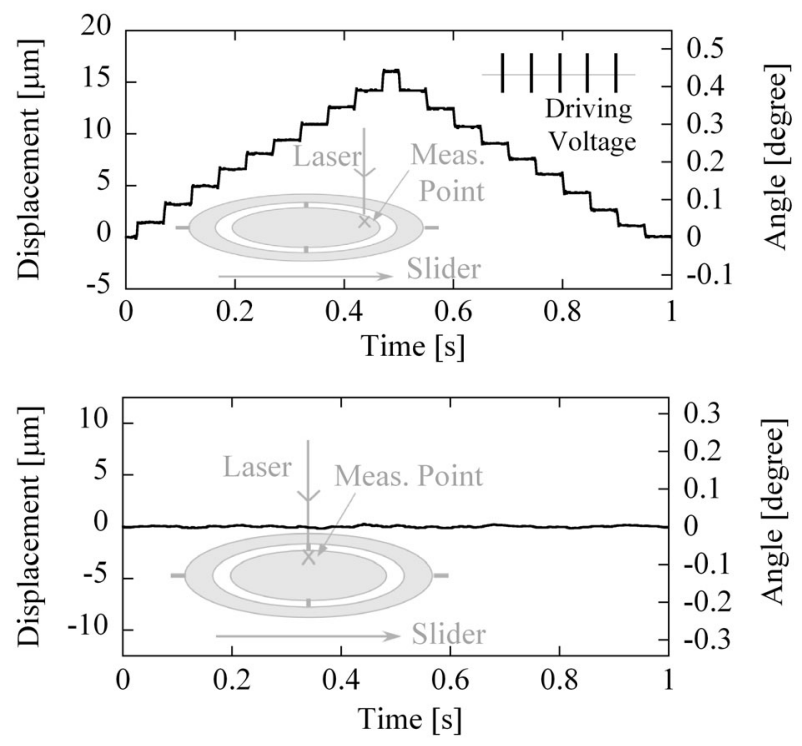

Fig. 7. The step motion of the mirror with an angle of 0.05-degree step and the displacement near the hinge of the gimbal; $50 \mathrm{~V}_{\text {peak }}$ driving voltage, 0.25 $\mathrm{ms}$ voltage applying duration, and $50 \mathrm{~ms}$ step period.

\section{References}

[1] M. Kurosawa, M. Takahashi, and T. Higuchi, "Ultrasonic Linear Motor Using Surface Acoustic Waves," IEEE Trans. Ultrason., Ferroelect., Freq. Contr., vol. 43, pp. 901-906, 1996.

[2] M. K. Kurosawa, "State-of-the-art surface acoustic wave linear motor and its future applications," Ultrasonics, vol. 38, pp. 15-19, 2000.

[3] T. Shigematsu, M. K. Kurosawa, and K. Asai, "Nanometer Stepping Drives of Surface Acoustic Wave Motor," IEEE Trans. Ultrason., Ferroelect., Freq. Contr., vol. 50, pp. 376-385, 2003.

[4] T. Shigematsu and M. K. Kurosawa, "Miniaturized SAW motor with $100 \mathrm{MHz}$ Driving Frequency," in Proc. 17th IEEE Int. Micro Electro Mechanical Systems Conf., pp. 482-485, 2004.

[5] T. Shigematsu and M. K. Kurosawa, "Nanometer resolution 2-D in-plane SAW motor," in Proc. 9th Int. Conf. New Actuators 2004, pp. 140-143, 2004.

[6] Y. Nakamura, M. K. Kurosawa, T. Shigematsu, and K. Asai, "Effects of Ceramic Thin Film Coating on Friction Surfaces for Surface Acoustic Wave Linear Motor," in Proc. IEEE Ultrasonics Symp., pp. 1766-1769, 2003.

[7] T. Iseki, T. Shigematsu, M. Okumura, T. Sugawara, and M. K. Kurosawa, "Two-dimensionally self-holding Deflector with SAW Actuation," in Proc. IEEE/LEOS Int. Conf. Optical MEMS, pp.62-63, 2004. 